\title{
AUTOMATIC ROOM CONDITIONING SYSTEM FOR MASS PRODUCTION OF FRUIT-FLY
}

\author{
Md. Atiar Rahman ${ }^{1 *}$, Md. Abdullah Al Mamun², Sabiha Sattar ${ }^{3}$, Mohaimina \\ Begum $^{4}$ and Anisa Begum 5 \\ 1,2,3,4,5Electronics Division, Atomic Energy Centre Dhaka, Bangladesh \\ 1*atiar.aecd@gmail.com, ${ }^{2}$ mamun.aec@gmail.com, ${ }^{3}$ sabihasattar@yahoo.com, \\ ${ }^{4}$ mohaimina@gmail.com, ${ }^{5}$ anisa82@dhaka.net
}

\begin{abstract}
This paper presents an automatic room conditioning system for controlling temperature and relative humidity at an essential level. The system is intended to design for the production of fruit-fly in a mass scale by automatically maintaining the room conditions of sterile insect technique (SIT) laboratory. The designed system consists of microcontrollers, a temperature and relative humidity sensor module, a radio-frequency ( $R F$ ) data transmitter and receiver module, keypad and switching circuits. The integrated system works as a microcontroller acquires the temperature and relative humidity value from the sensor module, compares it to the predefined value of these parameters, and generates corresponding signals for the switching circuits to keep the temperature and relative humidity at required level. Data acquisition from senor module is performed using bidirectional 2-wire communication protocol. Moreover, the temperature and relative humidity data along with switching records are transmitted to a remote monitoring unit using RF transmitter and receiver module, where the more flexible universal synchronous/asynchronous receiver/transmitter (USART) protocol is employed for performing the serial communication between microcontroller and RF transmitter and receiver modules. The results show that the developed prototype system is performing satisfactory to maintain the desired room conditioning for mass production of fruit-fly of SIT laboratory and to monitor the room conditions remotely. It is supposed that this low-cost user friendly system can also contribute in many research and industrial areas, where automatic room conditioning system is crucial.
\end{abstract}

Keywords - SHT75 sensor, Microcontroller, Automatic room conditioning system, Sterile insect technique, Fruit-fly

\section{INTRODUCTION}

In the applications of research laboratories and industrial process, it is an important requirement to keep room temperature and relative humidity at a desired level. In addition, it is beneficial to monitor the necessary data remotely, where the entry of the controlled area is restricted. A lot of works had been reported on the monitoring of temperature and relative humidity [1] - [7]. However, most of the works introduced only monitoring or measurement of temperature and relative humidity. For example, Hao and Zongtao designed a system for wireless monitoring of environmental temperature and humidity [1]. Cherukuri and Kanchi designed a system for integrated relative humidity, temperature measurement and gas (LPG) sensing system [2]. Some works were also

Received: July 4, 2019

Reviewed: October 15, 2019

Accepted: February 10, 2020

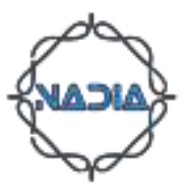


noticed only control of either temperature or humidity [7], [8]. Nevertheless, very few works were found on the automatic control of temperature and relative humidity mutually. Moreover, it was also observed that some systems employed separate sensors for temperature and relative humidity measurement [1], [3], which reduces design flexibility. Based on the literature review, the design of an automatic room conditioning system should be suggested that will perform the measurement, monitoring and controlling of temperature and relative humidity mutually using integrated sensor module.

The Sterile Insect Technique (SIT) Laboratory of Insect Biotechnology Division, Institute of Food and Radiation Biology (IFRB), Atomic Energy Research Establishment (AERE), Savar, Dhaka, Bangladesh, is involved in developing cost effective artificial diet for mass rearing of fruit-fly (melon fly), optimization of quality parameters and field cage trials for the application of SIT program in the designated areas of Bangladesh. To carry out the SIT program, the temperature and relative humidity of the rearing room should be maintained at $27( \pm 2){ }^{\circ} \mathrm{C}$ and $75( \pm 5) \% \mathrm{Rh}$ respectively [9]. However, it is observed that the temperature and relative humidity was monitored there by using a conventional thermometer and hygrometer. Moreover, the temperature and relative humidity controlling instruments such as air cooler (AC), room heater, and humidifier were switched ON or OFF manually by human-operator to keep the required level of temperature and relative humidity. Therefore, it was difficult to maintain the desired temperature and relative humidity for adequate production of melon fly by following the above procedure. As a result, production of melon fly was decreased. Also, movement of human being at rearing room may have negative impact for proper growth of fruit-fly. To remove these constrains, the design and development of automatic room conditioning system for mass production of fruit-fly is demanded.

Purpose of this present work is to automatically maintain the required temperature and relative humidity needed in rearing room of fruit-fly mass production at SIT laboratory. The designed system can maintain automatically the desired level of temperature and relative humidity at rearing room by sensing the temperature and relative humidity from the sensor and accordingly control the switching of three fundamental electrical apparatuses such as AC, room heater, and humidifier. In addition, the system has the facility to monitor these room conditioning parameters with switching information remotely that reduces the movement of human operator inside the rear room and is supposed to enhance the production of fruit-fly.

\section{SYSTEM ARCHITECTURE}

Fig. 1 demonstrates the design of the system architecture. It shows that the designed system has two parts- (a) Rearing Room Automatic Control Unit (RRACU) and (b) Remote Monitoring Unit (RMU).

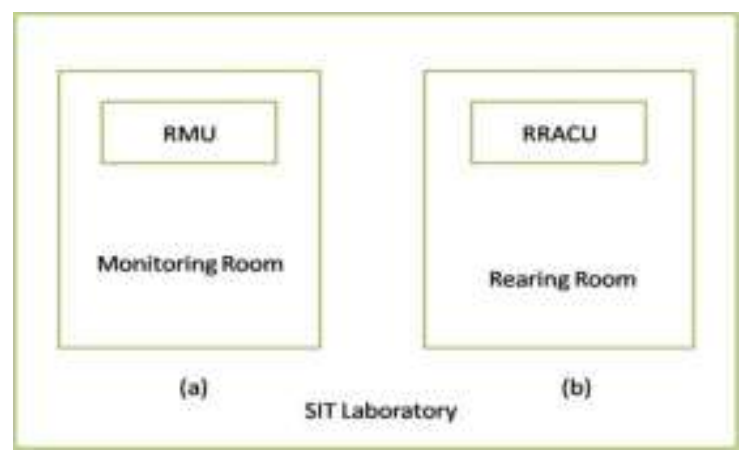

Fig. 1 System Architecture of Automatic Room Conditioning System at SIT Laboratory (a) RRACU and (b) RMU. 
The detail block diagram of these two parts is shown in Fig. 2. Fig. 2(a) shows that RRACU is the major part of the designed system, which includes an AVR series microcontroller ATMega-32, an integrated temperature and humidity sensor SHT-75, keypad, switching circuits and a RF transmitter module KST-TX01. SHT-75 provides the real-time temperature and relative humidity of the rearing room, which is compared with the previously set value entered with keypad and generates corresponding control signals for switching circuits by ATMega-32. Thus, it can keep the rearing room temperature and relative humidity at desired level automatically. It also sends the real-time temperature and relative humidity data along with switching status of the controlling equipments to the RMU through RF transmitter.

On the other hand, Fig. 2(b) shows RMU, which provides an option to the user for monitoring the condition of rearing room remotely. This unit receives the integrated data of temperature and relative humidity along with switching status sent from RRACU. For this, an ATMega-32 microcontroller is interfaced with a KST-RX806 RF receiver module to receive the transmitted data and a liquid crystal display (LCD) is employed to display the received data for the operator.

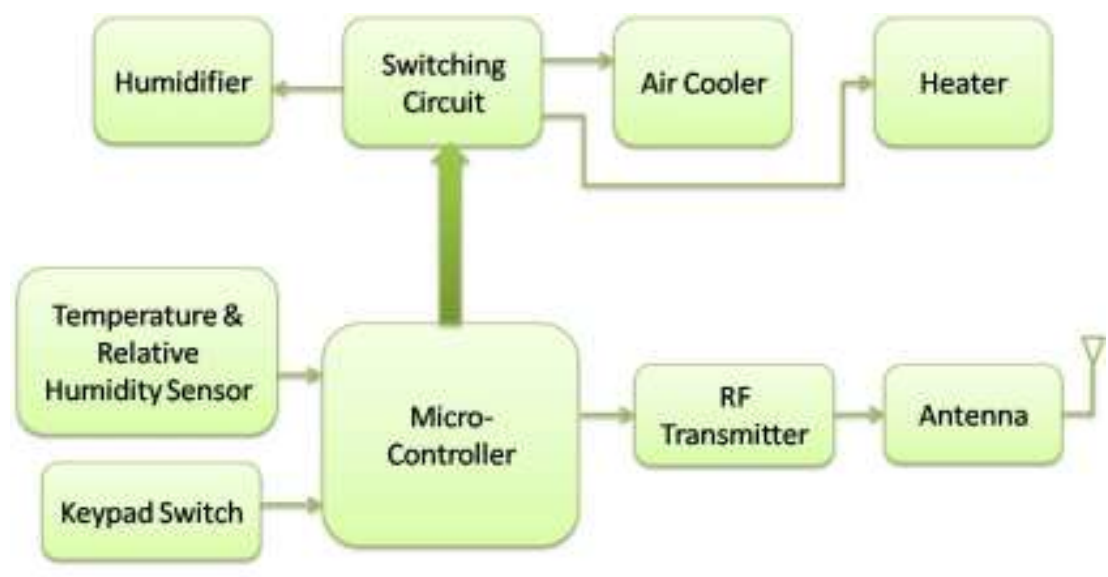

(a)

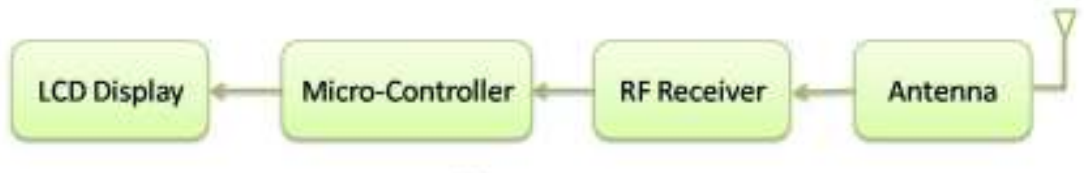

(b)

Fig. 2 Block diagram of (a) Rearing Room Automatic Control Unit (RRACU) and (b) Remote Monitoring Unit (RMU).

\section{CIRCUIT DIAGRAM, OPERATION AND DESCRIPTION}

Fig. 3 presents the detail schematic diagram of Rearing Room Automatic Control Unit (RRACU). It shows that RRACU consist of a keypad, a sensor, a switching circuit, a LCD display and a RF transmitter. Keypad is used to set the required temperature and relative humidity values so that microcontroller can understand and works accordingly. Keypad contains four keys- MODE, INCR, DCR and ENTER. MODE key provides option to the user to set temperature and relative humidity values as shown in Fig. 4(a) and (b). INCR and DCR keys are used to increase and decrease the values in order to get the exact values of input, then press the ENTER key to store these values. As we need to keep the temperature and relative humidity $27( \pm 2){ }^{\circ} \mathrm{C}$ and $75( \pm 5) \% \mathrm{Rh}$ respectively for mass production of fruit-fly [9], we entered the temperature and relative humidity at 27 ${ }^{\circ} \mathrm{C}$ and $75 \% \mathrm{Rh}$ respectively as demonstrated in Fig. 4 (c). 


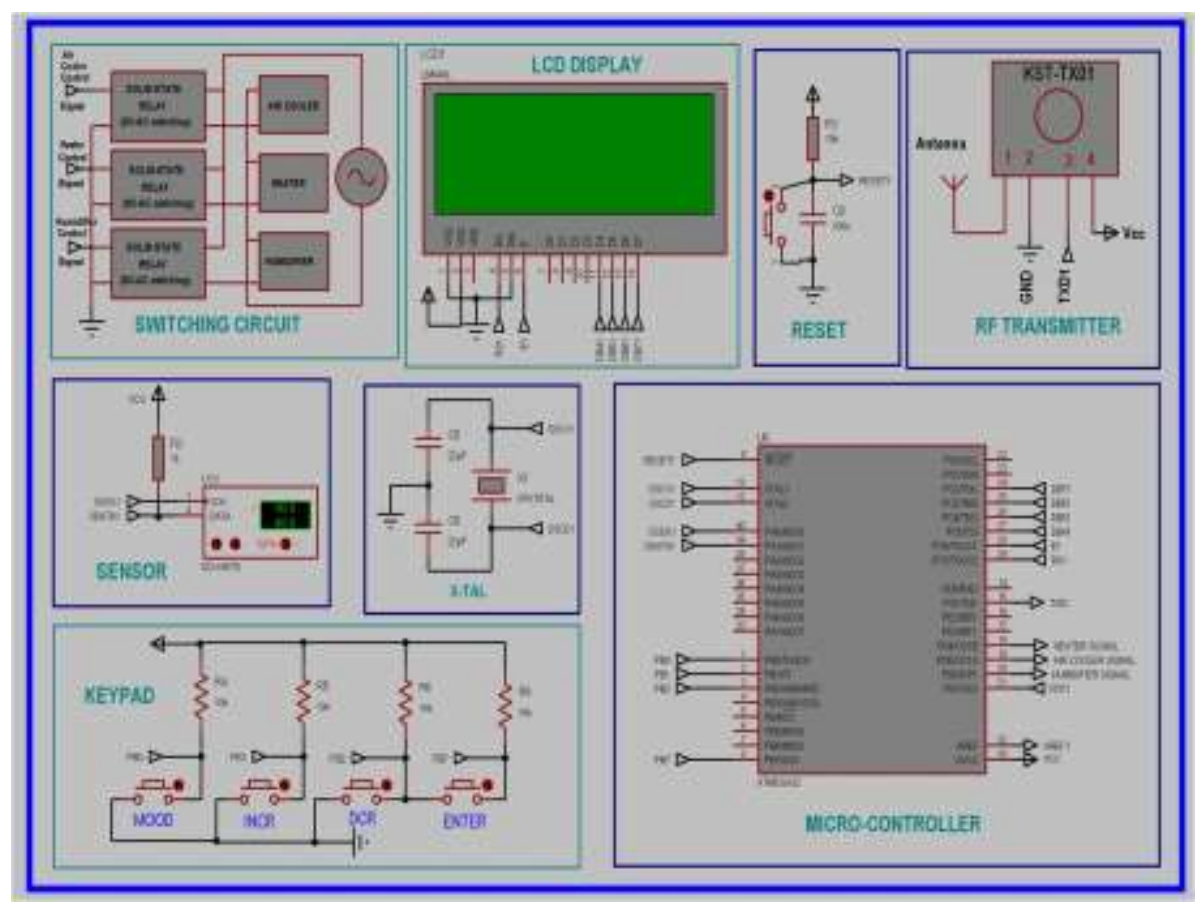

Fig. 3 Schematic diagram of Rearing Room Automatic Control Unit (RRACU).

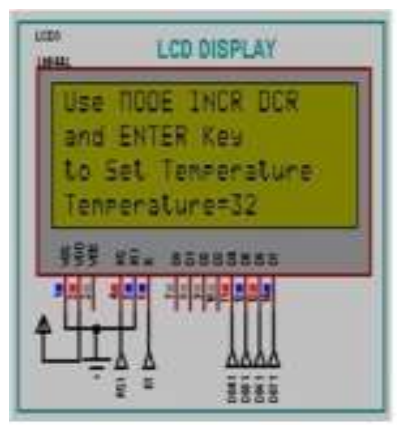

(a)

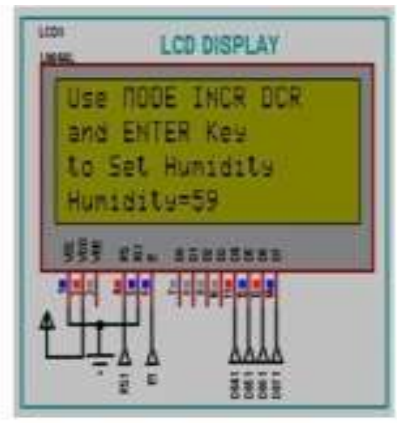

(b)

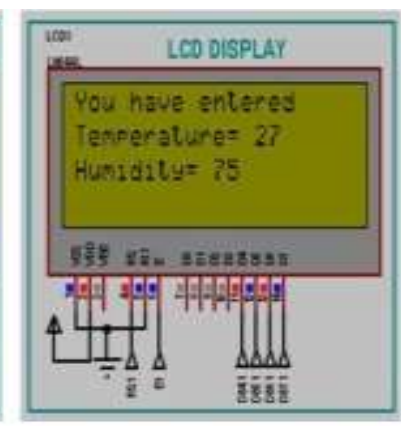

(c)

Fig. 4 Procedure to set input required (a) temperature, (b) relative humidity and (c) show the entered values using keypad

SHT75 is used as Temperature and Relative humidity sensor which is a SHT7x series (SHT71 and SHT75) Sensirion's family of relative humidity and temperature sensor. This type of sensor integrates sensor elements plus signals processing in compact format and provides a fully calibrated digital output. The digital data of the sensor is defined as 12 bit for $\% \mathrm{Rh}$ and 14 bit for temperature. The operating range is 0 to $100 \% \mathrm{Rh}$ and $-40^{\circ} \mathrm{C}$ to $123.8^{\circ} \mathrm{C}$ respectively for $\% \mathrm{Rh}$ and temperature, with a relative humidity accuracy of $\pm 2.0 \% \mathrm{Rh}$ and temperature accuracy of $\pm 0.4^{\circ} \mathrm{C}$. The interface between SHT75 sensor module and microcontroller is shown in Fig. 5(a). The sensor module has four pins. $V_{\mathrm{dd}}$ is the supply voltage to the sensor ranging from 2.4 to 5.5 Volt DC. SCK is the serial clock input to the sensor. SCK is used to synchronize the communication between microcontroller and SHT75. DATA is the serial data line which is a bidirectional communication pin between master (Microcontroller) and slave (SHT75). DATA changes after the falling edge and is valid on the rising edge of the serial clock SCK [10]. The timing diagram for valid data communication of the sensor module is shown in Fig. 5(b). 


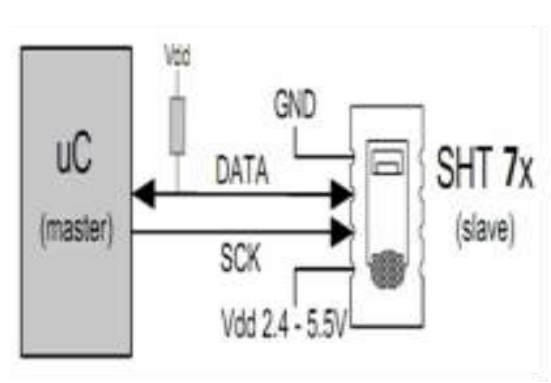

(a)

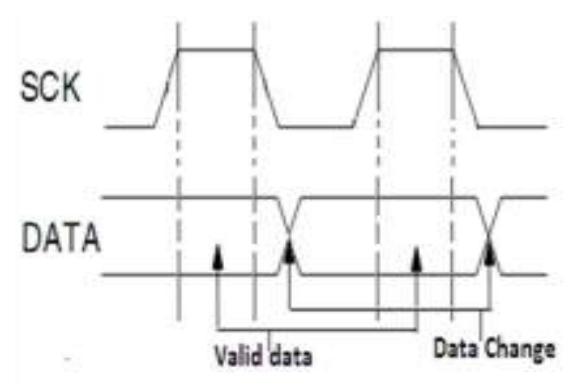

(b)

Fig. 5 (a) Interface circuit and (b) Timing diagram for valid data communication between sensor module and microcontroller.

To acquire temperature and relative humidity data from SHT-75 sensor, microcontroller (ATMega-32) initiates a transmission start command. After sending transmission start sequence the SHT75 sensor is ready to get data acquisition command ('00000011' for temperature and '00000101' for relative humidity) from ATMega-32. After issuing a data acquisition command ATMega-32 has to wait for the accomplishment of measurement. This takes a maximum of 20/80/320 ms for an $8 / 12 / 14$ bit measurement. Measured temperature and relative humidity data are now compared with the previously stored set temperature and relative humidity $\left(27^{\circ} \mathrm{C}\right.$ and 75 $\% \mathrm{Rh}$ ), to produce switching signal for air cooler, room heater and humidifier, respectively.

Since the rearing room temperature should be maintained at $27( \pm 2){ }^{\circ} \mathrm{C}$, the maximum and minimum temperature points can be reached at $29^{\circ} \mathrm{C}$ and $25^{\circ} \mathrm{C}$, respectively if the set value is $27^{\circ} \mathrm{C}$. Suppose that if the temperature becomes $25^{\circ} \mathrm{C}$, the heater is switched $\mathrm{ON}$ and remain ON until the temperature comes to $27^{\circ} \mathrm{C}$. At $27^{\circ} \mathrm{C}$, the heater is switch OFF and remains OFF until temperature returns back to $25^{\circ} \mathrm{C}$. At $29{ }^{\circ} \mathrm{C}$, the $\mathrm{AC}$ is switched $\mathrm{ON}$ and remains $\mathrm{ON}$ until the temperature comes to $27^{\circ} \mathrm{C}$. At $27^{\circ} \mathrm{C}$, the $\mathrm{AC}$ is switch OFF and remains OFF until temperature returns back to $29{ }^{\circ} \mathrm{C}$. The temperature dependent control signal for AC and heater is shown in Fig. 6(a).

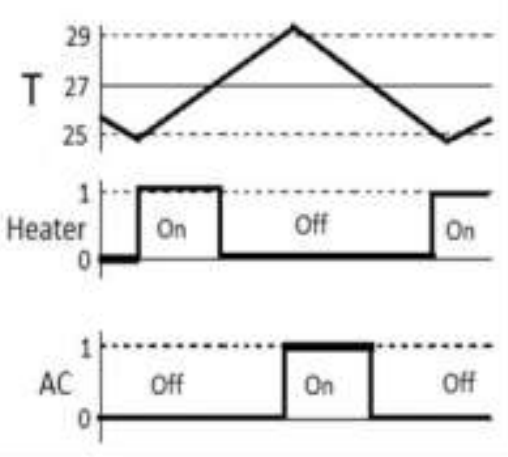

(a)

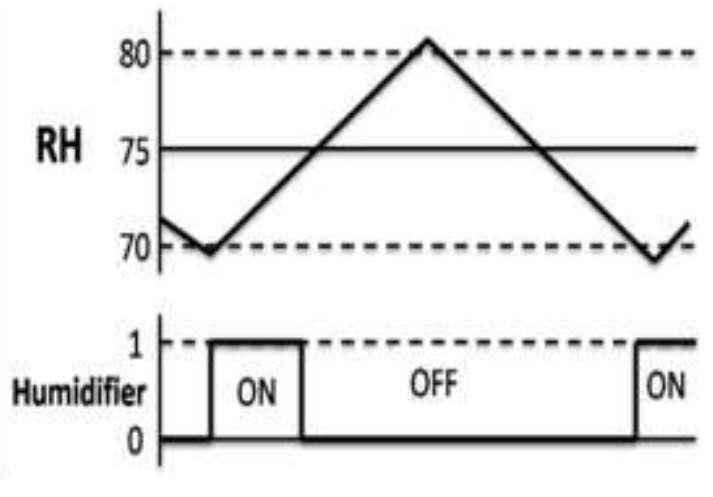

(b)

Fig. 6 Control signal for (a) AC and Heater and (b) for Humidifier

Similarly, the rearing room relative humidity should be maintained at $75( \pm 5) \% \mathrm{Rh}$ (Khan et al., 2007), the maximum and minimum humidity point can be reached $80 \% \mathrm{Rh}$ and $70 \% \mathrm{Rh}$, respectively if the set value is $75 \% \mathrm{Rh}$. Suppose that if the humidity becomes $70 \% \mathrm{Rh}$, the humidifier is switched $\mathrm{ON}$ and remains $\mathrm{ON}$ until the humidity reaches at $75 \% \mathrm{Rh}$. At $75 \% \mathrm{Rh}$, the humidifier is switched OFF and remains OFF until 
humidity returns back to $70 \% \mathrm{Rh}$. As the maximum average humidity of Dhaka, Bangladesh [11] is not more than $75 \% \mathrm{Rh}$, it is suggested that the use of dehumidifier in the designed system is not mandatory. The relative humidity dependent control signal for humidifier is shown in Fig. 6(b). Intentionally, the intermittent OFF time of heater, AC and humidifier has been intelligently controlled compared to conventional so that it can save the energy and enhance the system efficiency.

Control signal generated from microcontroller is supplied to the switching circuit which consists of solid state relay (SSR) and circuit breaker. SSR is a switching device having no moving parts that contains two terminals- input terminal and output terminal. A low signal is applied to the input terminal of SSR to drive a high load (AC/DC) at output terminal. Since, air cooler, heater and humidifier are the instruments of high current rating, switching circuit uses FOTEK SSR-40DA solid state relay that is capable of driving current loads of up to 40A with a 3-32V DC input. For additional safety, separate circuit breakers are used for each control equipment. Finally, KST-Tx-01 wireless RF transmitter module is used by RRACU to send the temperature and relative humidity along with switching status of AC, heater and humidifier to RMU. An LCD display is also available at RRACU for visualization of necessary setting parameters to the system operator.

Fig. 7 shows the schematic diagram of Remote Monitoring Unit (RMU). Transmitted data from RRACU is received by an antenna to the RF receiver module at RMU. KST$\mathrm{RX806}$ wireless data receiver module is used as RF receiver. This RF module is also interfaced with an AVR series ATMega-32 microcontroller. Data reception at RMU is performed using serial communication protocol. After successful data reception, the microcontroller displays the data to a LCD display.

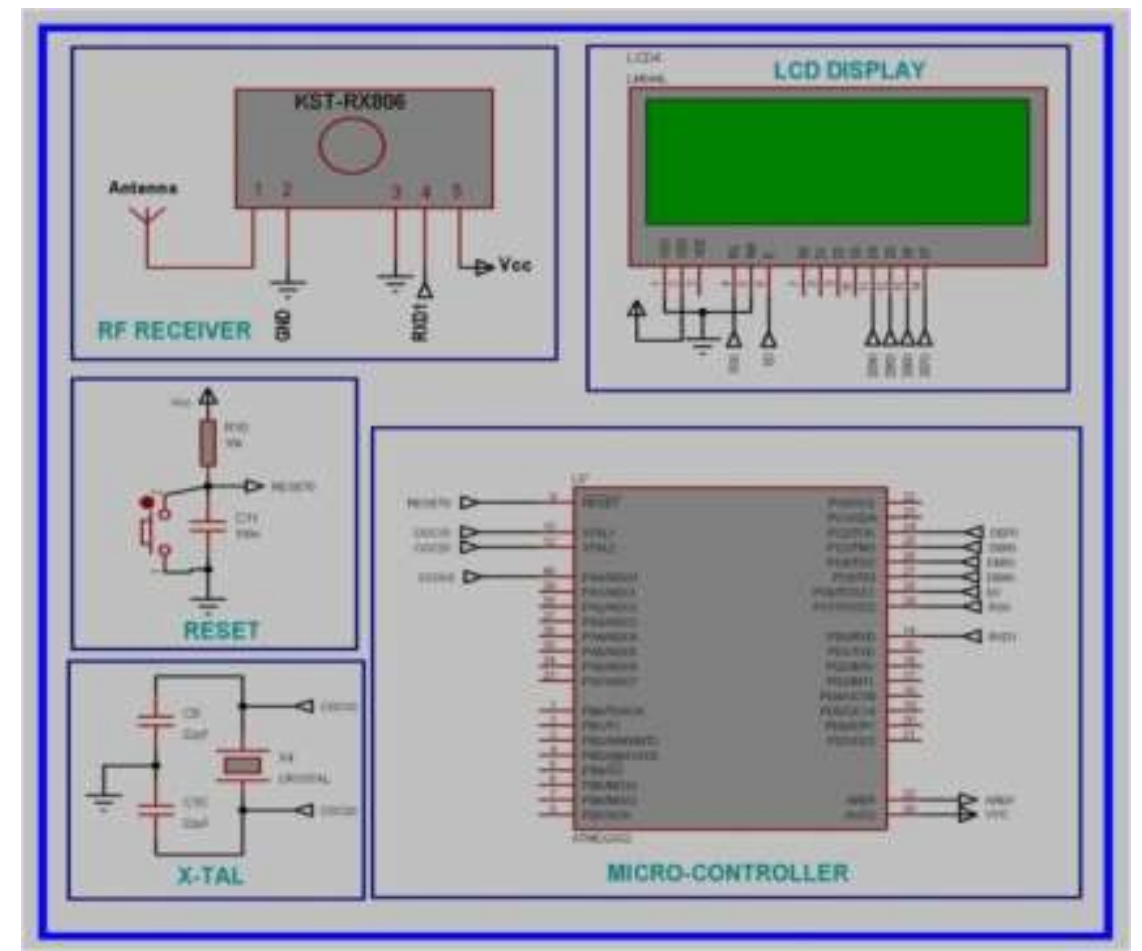

Fig. 7 Schematic diagram of Remote Monitoring Unit (RMU)

BASCOM AVR software is used to write firmware code for ATMega-32 microcontrollers used in both RRACU and RMU module. Then the desired HEX file is generated using same software. The generated HEX file is loaded to the microcontrollers 
using universal programmer. Program flowchart for RRACU and RMU are shown in Fig. 8 (a) and (b), respectively.

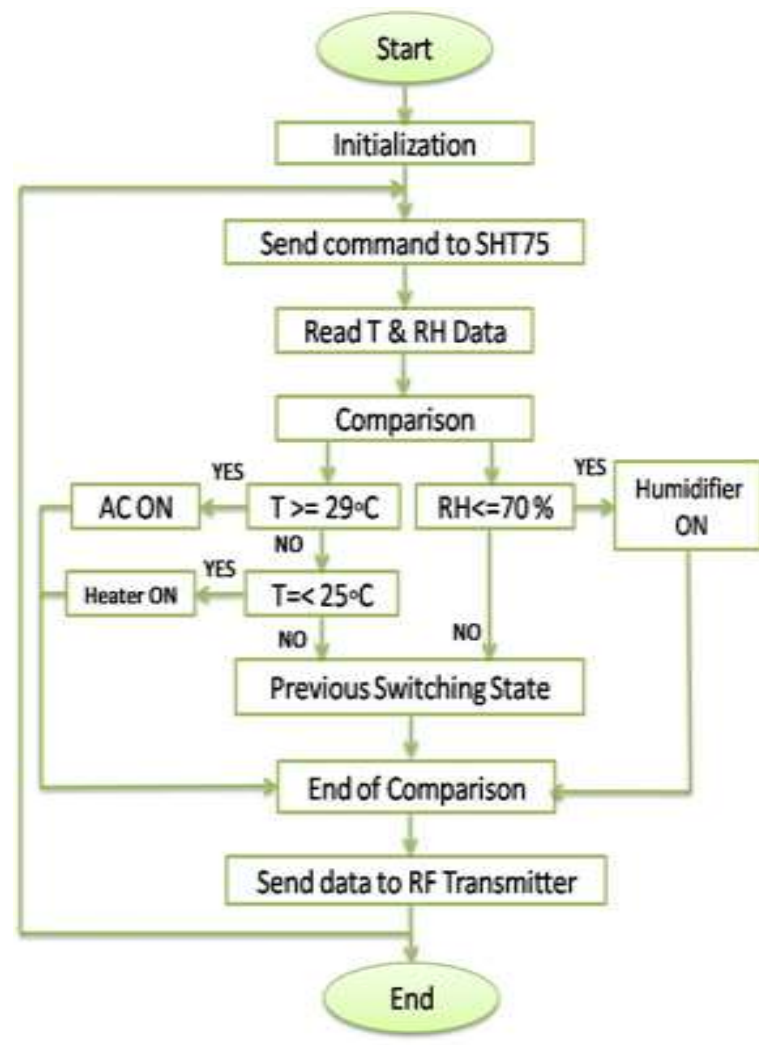

(a)

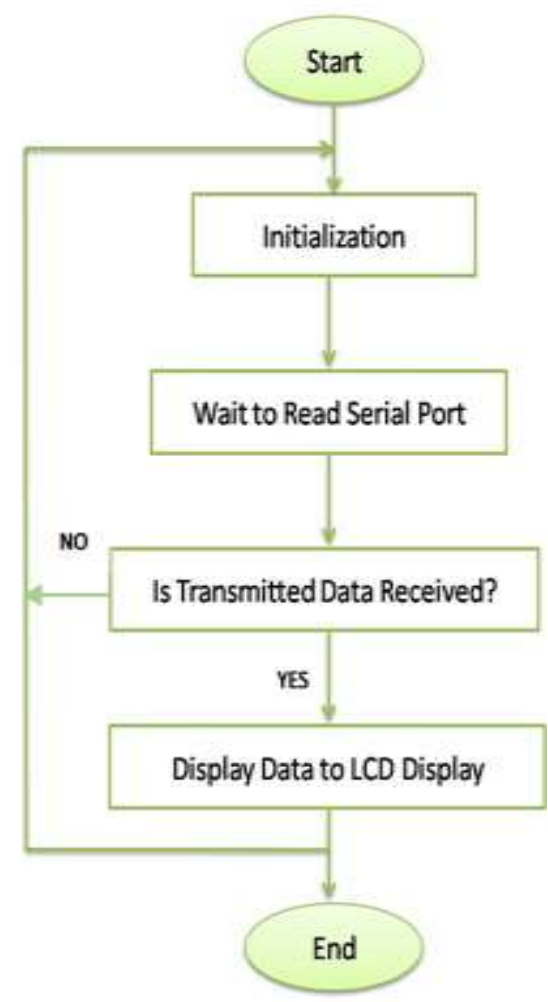

(b)

Fig. 8 Program flow chart for (a) RRACU and (b) RMU

\section{RESULTS AND DISCUSSION}

A laboratory grade prototype of the complete system has been developed and installed at SIT laboratory as shown in Fig. 9. It was found that the system was working properly and able to keep the desired temperature and relative humidity automatically. The operation of the system was demonstrated by the display of LCD monitor. Fig. 10 (a) shows a model display of output data from RRACU, where measured temperature is higher and relative humidity is lower than the previously inputted set values of them. This Fig. also shows the switching status of $\mathrm{AC}$, heater and humidifier, respectively. It is seen that measured temperature is $30{ }^{\circ} \mathrm{C}$, whereas the set temperature is $27{ }^{\circ} \mathrm{C}$. In this condition, heater should be switched OFF, and $\mathrm{AC}$ should be switched $\mathrm{ON}$ as per conditions defined in Fig. 8(a). Furthermore, the measured humidity is $61.49 \% \mathrm{Rh}$ and the set humidity is $75 \% \mathrm{Rh}$. With this condition, humidifier should be switched ON. Fig. 10(b) shows the similar data of Fig. 10(a) received at RMU, which indicates the satisfactory data transmission between RRACU and RMU. 


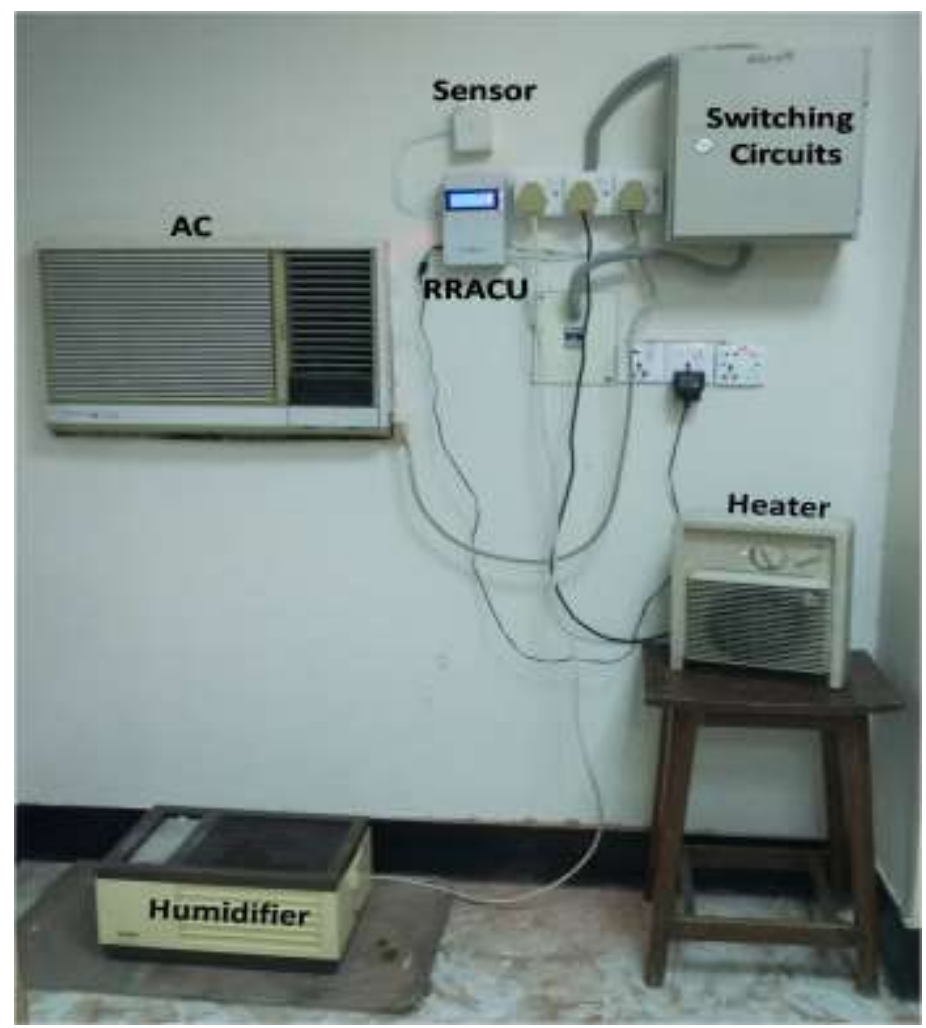

Fig. 9 Photographs of the developed and installed system at SIT laboratory

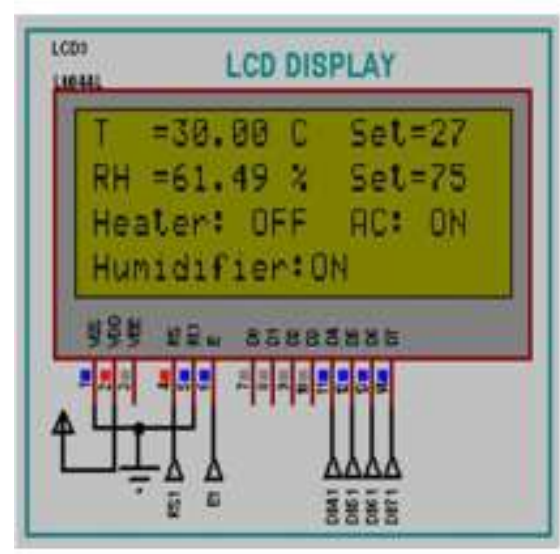

(a)

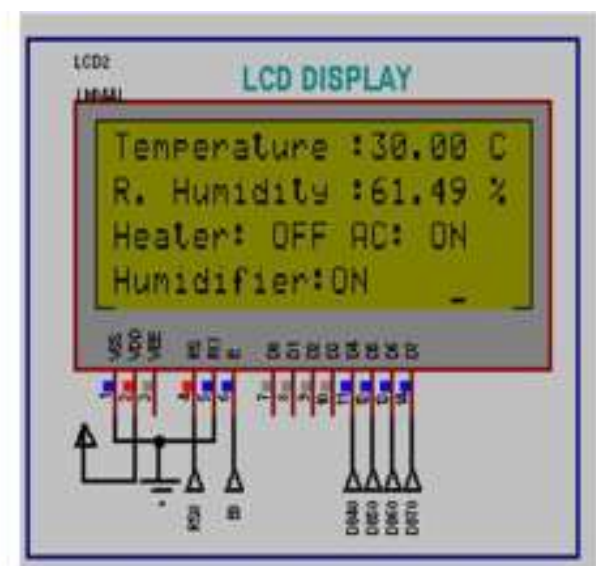

(b)

Fig. 10 LCD display of output data at conditions of measured temperature is higher and relative humidity is lower than set values for (a) RRACU and (b) RMU.

Furthermore, Fig. 11(a) and (b) shows a model display of output data from RRACU and RMU respectively, where measured temperature is lower and relative humidity is higher than the previously inputted set values of them. It is seen that measured temperature and relative humidity are $23.99{ }^{\circ} \mathrm{C}$ and $75.43 \% \mathrm{Rh}$, whereas the set values are $27{ }^{\circ} \mathrm{C}$ and $75 \% \mathrm{Rh}$, respectively. In this condition, AC should be switched OFF, heater should be switched ON and humidifier should be switched OFF, which satisfies the displayed status of Fig. 11. 


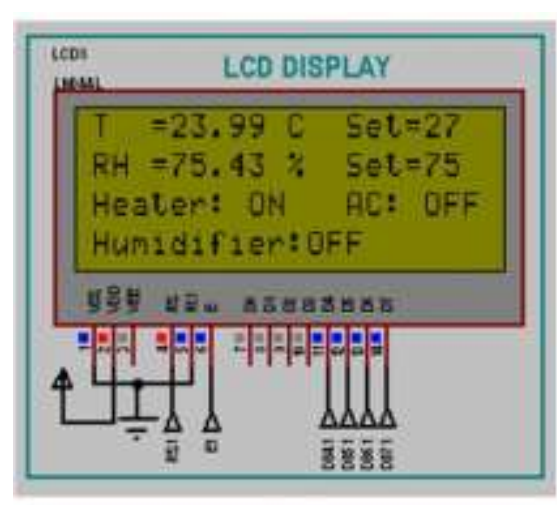

(a)

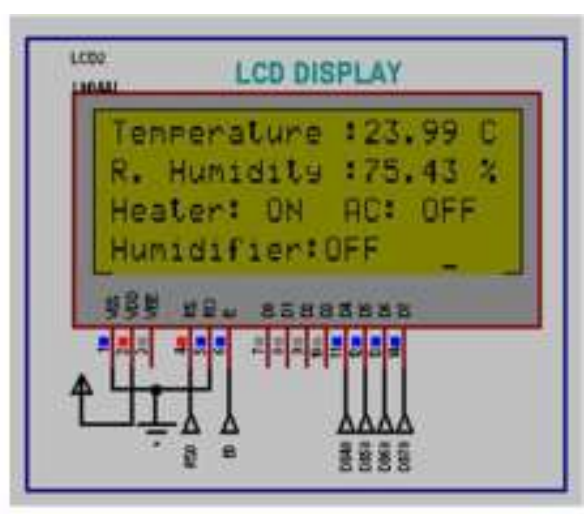

(b)

Fig. 11 LCD display of output data at conditions of measured temperature is lower and relative humidity is higher than set values for (a) RRACU and (b) RMU.

These results show that the designed system is capable to maintain the predefined temperature and relative humidity at required level, thus providing a satisfying automatic room conditioning system. This in turn provides a great improvement in mass production of fruit-fly observed by the operators at SIT laboratory. The operators also identified that the RMU provides a disturb free monitoring interface for viewing the status of the rearing room. However, in future, a programmable light intensity controller can be incorporated with the existing system to create an artificial day-night environment to carry out further research on fruit-fly rearing at SIT laboratory as per demanded by the operators.

\section{CONCLUSIONS}

In this paper, an automatic room conditioning system has been introduced for controlling temperature and relative humidity at a required level for the mass production of fruit-fly at SIT laboratory. The developed system mainly highlights the functional characteristics of microcontroller with temperature and humidity sensor, switching circuits, remote data monitoring, low cost production and user friendly operation. The system has also been found energy efficient as the switching time of heater, AC and humidifier is controlled intelligently compared to conventional approach. The tested results from the system offer real-time measurement accuracy and good outcomes in mass production of fruit-fly at SIT laboratory. This developed low cost system is supposed to be may contribute in many research and industrial areas such as food processing, incubator, cold storage and so on.

\section{ACKNOWLEDGMENTS}

The authors would like to thank the Sterile Insect Technique(SIT) Laboratoryof Insect Biotechnology Division, Institute of Food and Radiation Biology (IFRB), Atomic Energy Research Establishment (AERE), Savar, Dhaka, Bangladesh, for the support to carry out this research work.

\section{REFERENCES}

[1] Sun Hao and Chi Zongtao, "Design of the environmental temperature and humidity wireless monitoring system," 2015 12th IEEE International Conference on Electronic Measurement \& Instruments (ICEMI), Qingdao, 2015, pp.1652-1657. doi: 10.1109/ICEMI.2015.7494529

[2] Rajeswari cherukuri and Raghavendra rao kanchi. "Design and Development of an Integrated Relative Humidity, Temperature Measurement and Gas(LPG) Sensing System Using PIC18F25K20" Research \& Reviews: Journal of Pure and Applied Physics 3.1 (2015): 1-7. URL: https://pdfs.semanticscholar.org/b6a9/83808ed5a5ee5e856d93bf77902cac584776.pdf 
[3] T. Bheema lingaiah, D.Hanumesh Kumar, C.Nagaraja, Solomon Woldetsadik. "Development of Humidity and Temperature Measurement Instrumentation System using LabVIEW" International Journal of Advanced Research in Electrical, Electronics and Instrumentation Engineering 2.10 (2013): 6365-6372. URL: https://www.ijareeie.com/upload/2013/december/2C_Development.pdf

[4] M. Simić, "Design and development of air temperature and relative humidity monitoring system with AVR processor based web server," 2014 International Conference and Exposition on Electrical and Power Engineering (EPE), Iasi, 2014, pp.038-041. doi: 10.1109/ICEPE.2014.6969864

[5] V. D. Bachuwar, A. D. Shligram, P. Prabhakarc and L. P. Deshmukhd. "Lab View Based Wireless Soil Moisture and Temperature Monitoring System for Water Irrigation Management" Indian Journal of Scientific Research 15.2 (2017): 186-190. URL: https://www.ijsr.in/upload/2012212285Chapter_32.pdf

[6] Balachandran, T \& Sbenaty, Saleh \& Walck, Jeffrey. "Remote humidity and temperature real-time monitoring system for the study of the after-ripening process in seeds" 120th ASEE Annual Conference and Exposition, Conference Proceedings. 23-26 June, 2013. URL: https://www.researchgate.net/publication/287268160_Remote_humidity_and_temperature_realtime_monitoring_system_for_the_study_of_the_after-ripening_process_in_seeds

[7] Dr. B. Ramamurthy, S. Bhargavi, Dr. R. Shashi Kumar. "Development of a Low-Cost GSM SMSBased Humidity Remote Monitoring and Control system for Industrial Applications" International Journal of Advanced Computer Science and Applications (IJACSA) 1.4 (2010): 20-26. URL: https://pdfs.semanticscholar.org/e13a/d21a419296ddd9fe02da4cf4d4f3cf6786d9.pdf

[8] S. Arutselvi, Sarah Maria Louis, S. Srinithi. "Monitoring and Control of Relative Humidity in Soil using LabVIEW" International Journal of Engineering Trends and Technology (IJETT) 9.10 (2014): 497-500. URL:https://pdfs.semanticscholar.org/f385/937d2c100cda2648766152d392eb91322e1c.pdf?_ga=2.501 $17088.775758536 .1562128408-910036283.1557995567$

[9] Mahfuza Khan, M. Aftab Hossain, M. Abdul Alim, M. Saidul Islam and Shakil A. Khan. "Management of fruit fly using Sterile Insect Technique" 7th Biennial Conference, Bangladesh Entomological Society, 29 December 2007, BRAC Auditorium, Dhaka.

[10] Datasheet: SHT7x (SHT71, SHT75) Humidity and Temperature Sensor IC, http://www.mouser.com/ds/2/682/Sensirion_Humidity_SHT7x_Datasheet_V5-469726.pdf

[11] https://www.weather-atlas.com/en/bangladesh/dhaka-climate 